\title{
Comportement hydraulique de deux bras de la Loire (Pirmil et la Madeleine) à Nantes.
}

\author{
P. Fattal :Institut de Géographie - Université de Nantes. \\ M. Sanchez, Y. Delanoë : Laboratoire de Mécanique et Géomécanique - \\ Université de Nantes.
}

\section{Résumé}

La Loire s'écoule dans ses deux bras actuels, la Madeleine et Pirmil avec un débit communément admis dans les proportions suivantes : $1 / 3$ pour le premier et $2 / 3$ pour le second. Deux séries complètes de mesures nous conduisent à penser qu'en dehors des périodes de crues, le partage des débits entre les deux bras aussi bien en flot qu'en jusant est proche de $50 \%$. Ce constat à une influence notable sur les matières en suspension, la morphologie du fond, les rives et les infrastructures.

\section{Introduction}

L'histoire de la Loire tant dans sa partie ligérienne que fluviale est ponctuée d'aménagements successifs visant à "dompter ses excềs" à des fins d'urbanisation, d'activité industrielle ou portuaire, de pêche, d'agriculture et de tourisme.

Au XVIII ème siècle, le secteur dans sa traversée nantaise se caractérisait par des îles et levées. La "Venise de l'ouest" obéissait alors à un régime fluvial et ce ne sont que les travaux décrêtés au cours des siècles qui ont étendus l'influence maritime. Plus tard, entre 1926 et 1950 les premiers comblements ont eu lieu et les raisons invoquées par l'administration étaient:

- le maintien de la stabilité des ouvrages menacés par l'abaissement du niveau de l'eau (dû aux extractions de granulats) et plus spécialement la stabilisation des quais empruntés par la voie ferrée,

- le désengorgement des rues,

- la suppression des bras non navigables dans le but de conférer à Nantes sa vocation portuaire.

Ces travaux achevés, conjugués à ceux ultérieurs, notamment l'arasement d'un haut-fond rocheux en amont de Nantes (le seuil de Bellevue) et le déplacement du chenal de la rive droite vers la rive gauche; la Loire s'écoule dans ses deux bras actuels, la Madeleine et Pirmil. II s'agissait pour nous de contribuer à demêler l'écheveau de l'impact des aménagements difficile à évaluer. Encore aujourd'hui, de nombreux désordres liés à l'action anthropique sont observés dans notre secteur d'érude.

\section{Les campagnes de mesures}

Nous effectuons depuis 1992 des campagnes de levés et et de mesures sur site. C'est en juillet et septembre 1994, puis en mai 1995 (S. Tran Van Thong, 1995)(10) que nous avons axé nos recherches sur la différence de débit qui pouvait exister entre les deux bras. Par ailleurs, nous avons procèdé à des prélèvements à la bouteille afin de localiser le bouchon vaseux au moment des campagnes, dans le but de dresser pendant les jours considérés des graphiques d'isoconcentrations. 


\begin{tabular}{|c|c|c|}
\hline Dates & Débits & Coef ficients de marée \\
\hline 5 juillet 94 & $440 \mathrm{~m}^{3} / \mathrm{s}$ & $47-50$ \\
\hline 7 septembre 94 & $240 \mathrm{~m}^{3} / \mathrm{s}$ & $107-108$ \\
\hline 13 et $14 \mathrm{mai} 95$ & $627 \mathrm{~m}^{3} / \mathrm{s}$ & $92-97$ \\
\hline
\end{tabular}

Tableau 1 : Conditions hydrauliques

Pour les campagnes, nous avons mobilisé deux embarcations, équipées de courantomètres (Braystoke et un modèle DCN3-NBA) ainsi que des bouteilles à prélèvements d'eau (pour les matières en suspension) sur la tranche d'eau susceptible de nous intéresser.

Le mode opératoire est le suivant:

Chacune des embarcations est positionnée en trois points (figure 1), dans chacun des bras, les appareils de mesures sont mouillés tous les mètres, jusqu'au fond. Les courantomètres, fournissent des indications sur la mesure instantannée du courant ainsi que sa direction; les bouteilles permettent quant à elles de récupérer des matières en suspension en vue d'analyses en laboratoire. L'inclinaison des câbles du fait du courant n'excède pas les 10 degrés.

Les mesures de courant et les prélèvements de matières en suspension sont effectués simultanément dans les deux bras, en chacun des points et une fois toute les heures.

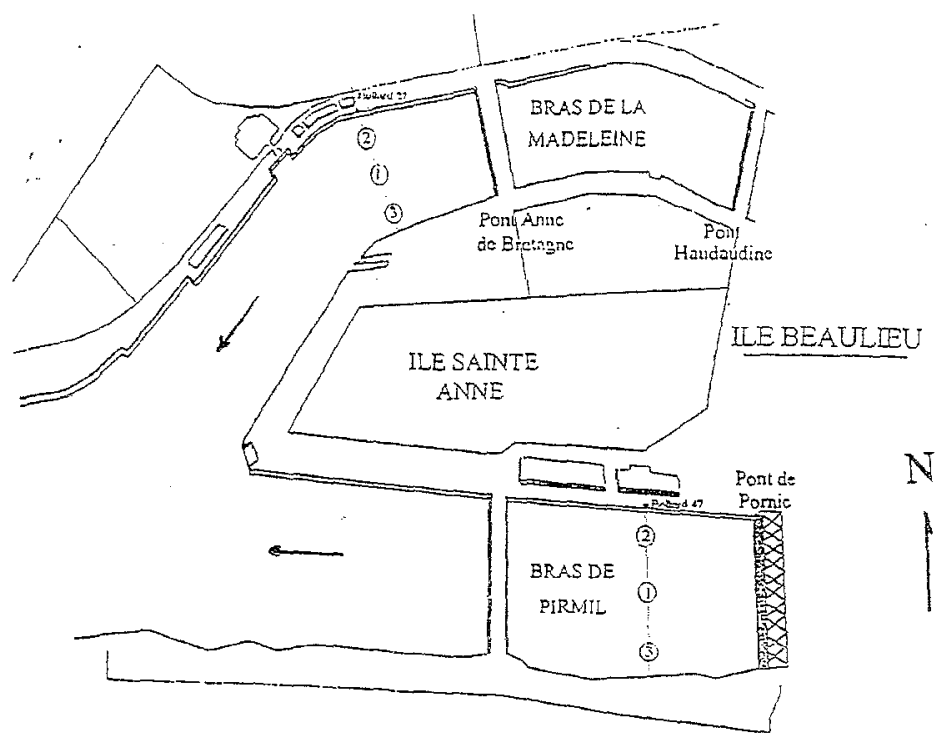

flgure 1 : carte d'implantation des mesures

(d'après (10)

\section{Méthode et résultats}

\subsection{Méthode}

A partir des mesures de courants, on calcule les volumes oscillants dans chaque bras durant les flot et le jusant et l'on obtient le volume oscillant global durant pour le cycle de marée. 
Dans chaque bras, les courants sont mesurés sur trois colonnes d'eau d'une section donnée, toutes les heures. Le calcul se déroule en trois phases:

Pour chaque profil de vitesse, on calcule le débit surfacique $D$ (intégration des vitesses à la verticale de chaque point à un instant donné, sur la profondeur).

en (i) à t

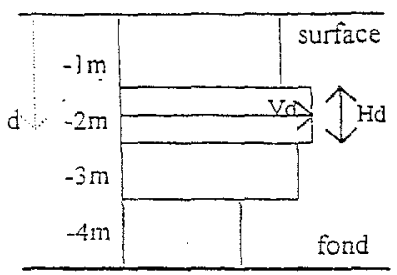

$$
D_{i}^{i}=\sum_{i} V_{d}^{i} \cdot H_{d}
$$

Hd étant la distance encadrant la profondeur d

Dans un second temps, on intègre les débits surfaciques de chaque point sur toute la période du flot et du jusant en supposant que le débit surfacique instantané est valable pendant la moitié de la durée qui sépare les deux heures de mesures encadrant l'heure considérée. On obtient la surface déflacée $S$ avec :

Aupoin (i)

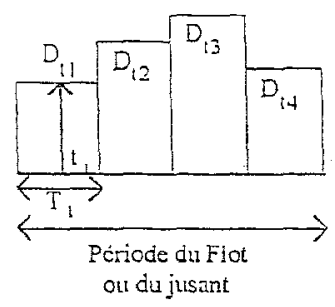

$$
S^{i}=\sum_{i} D_{1}^{i} \cdot T_{t}
$$

$T_{t}$ étant l'intervalle de temps encadrant l'instant, $t$

Pour calculer enfin le volume total déplacé, on multiplie-les surfaces précédentes par la largeur du fleuve correspondant aux points de mesures.

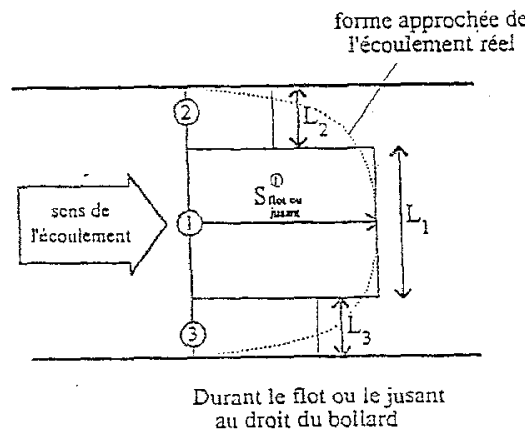

$$
V_{\substack{\text { Ros ou } \\ \text { jusant }}}=\sum_{i} S_{\text {jusant }}^{i \text { fou ou }} L^{i}
$$

Pour le bras de la Madeleine dont la largeur est d'environ 150 mètres, on prend : $\mathrm{L} 1=90 \mathrm{~m}, \mathrm{~L} 2=30 \mathrm{~m}, \mathrm{~L} 3=30 \mathrm{~m}$.

En ce qui concerne le bras de Pirmil, la rive gauche étant plus pentue, il est plus difficile d'estimer la largeur du bras. On a pris en compte la largeur moyenne entre la pleine-mer et la basse-mer : $\mathrm{L}=210 \mathrm{~m}$. Pour approcher la forme de l'écoulement, on prend : $\mathrm{L} 1=150 \mathrm{~m}, \mathrm{~L} 2=20 \mathrm{~m}$ er $\mathrm{L} 3=40 \mathrm{~m}$. 


\subsection{Résulitats}

BRAS DE LA MADELEINE BRAS DE PIRMIL

\begin{tabular}{|c|c|c|c|c|c|}
\hline & 1994 & 1995 & 1994 & 1995 \\
\hline $\begin{array}{c}\text { volume } \\
\text { déplacé } \\
\text { en millions } \\
\text { de } \mathrm{m}^{3}\end{array}$ & $\begin{array}{c}\text { durant } \\
\text { le flot }\end{array}$ & 15,4 & 8,9 & 16,9 & 10,2 \\
\cline { 2 - 6 } & $\begin{array}{c}\text { durant } \\
\text { le jusant }\end{array}$ & 22,1 & 23,6 & 24,2 & 24,8 \\
\cline { 2 - 7 } & $\begin{array}{c}\text { apports } \\
\text { liquidesdu } \\
\text { fleuve }\end{array}$ & 6,7 & 14,7 & 7,3 & 14,6 \\
\hline Débit & résiduel & $151 \mathrm{~m}^{3} / \mathrm{s}$ & $328 \mathrm{~m}^{3} / \mathrm{s}$ & $166 \mathrm{~m}^{3} / \mathrm{s}$ & $325 \mathrm{~m}^{3} / \mathrm{s}$ \\
\hline
\end{tabular}

tableau 2 : les résultats

Les résultats concement les volumes déplacés et les débits de chaque bras pour les années considérées. La somme des deux débits résiduels de chacun des bras doit coîncider avec le débit mesuré à la station de Montjean. En fait, il existe une légère différence $\left(77 \mathrm{~m}^{3} / \mathrm{s}\right.$ pour 94 et $26 \mathrm{~m}^{3} / \mathrm{s}$ pour 95$)$. Cet écart donne un indice de la précision des estimations (erreur au maximum de 5\% dans le calcul des volumes déplacés). Compte tenu d'une part du rôle régulateur du bassin de marée en amont de Nantes qui peut entraîner une différence entre le débit résiduel et le débit du fleuve, d'autre part de l'importance des volumes oscillants, on peut affirmer que les estimations approchent assez bien la réalité.

BRAS DE LA MADELEINE

\begin{tabular}{|c|c|c|c|c|c|}
\hline & \multirow{2}{*}{\multicolumn{2}{|c|}{1094}} & \\
\hline & & & & 1994 & 1995 \\
\hline \multirow{3}{*}{$\begin{array}{c}\text { volume } \\
\text { déplacé } \\
\text { en millions } \\
\text { de } \mathrm{m}^{3}\end{array}$} & $\begin{array}{l}\text { durant } \\
\text { le flot }\end{array}$ & $47,7 \%$ & $46,6 \%$ & $52,3 \%$ & $53,4 \%$ \\
\hline & $\begin{array}{c}\text { durant } \\
\text { le jusant }\end{array}$ & $47,7 \%$ & $48,8 \%$ & $52,3 \%$ & $51,2 \%$ \\
\hline & $\begin{array}{l}\text { apports } \\
\text { liquidesdu } \\
\text { fleuve }\end{array}$ & $47,8 \%$ & $50 \%$ & $52,2 \%$ & $50 \%$ \\
\hline Débit & résiduel & $151 \mathrm{~m}^{3} / \mathrm{s}$ & $328 \mathrm{~m}^{3} / \mathrm{s}$ & $166 \mathrm{~m}^{3} / \mathrm{s}$ & $325 \mathrm{~m}^{3} / \mathrm{s}$ \\
\hline
\end{tabular}

tableau $3:$ répartition des volumes oscillants en $\%$

\section{Anaivse hrdrologique}

\section{1. volumes oscillants et débits}

Le tableau des résultats montre que les volumes d'eau sont plus importants dans le bras de Pirmil, pendant le flot et le jusant. Celà provient de sa section moyenne plus importante. Par contre, la répartition des volumes oscillants n'est pas le même au flot et au jusant (figure 2). En effet, c'est au jusant que le partage est plus équilibré. Il semblerait que l'arasement du seuil de Bellevue ait influencé ce partage des eaux du jusant par une modification des conditions d'écoulement. 


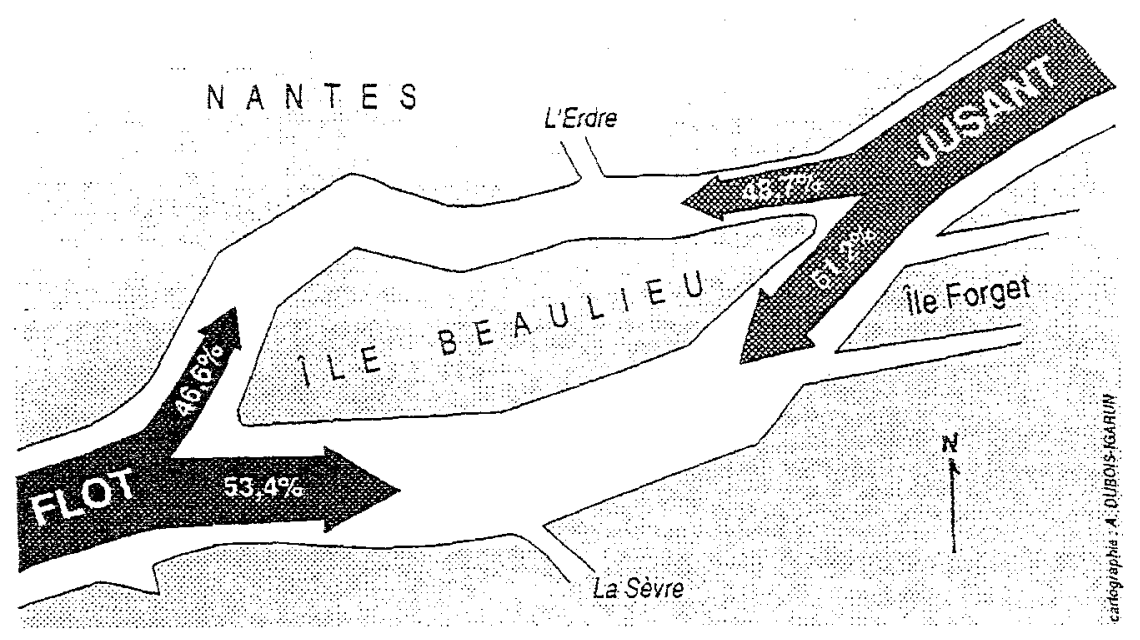

figure 2 : volumes oscillants de flot et de jusant en 1995

(d'après (10)

Même si les quantités d'eau déplacées sont plus importantes dans le bras de Pirmil, on observe que sur le cycle de la marée, les apports liquides du fleuve sont équivalents dans chaque bras. La répartition des débits en crue peut à priori être différente de celle en débit moyen par suite d'un écoulement plus turbulent ou d'une variation de la section mouillée plus importante pour le bras de Pirmil que pour le bras de la Madeleine (le bras de Pirmil sur sa rive sud n'est pas bordé par des quais contrairement à celui de la Madeleine). En période de crue (à partir de $2000 \mathrm{~m}^{3} / \mathrm{s}$ ), il est probable que le jusant prédominerait sur le flot. La répartition des apports liquides du fleuve devrait alors se rapprocher de $51 \%$ pour le bras de Pirmil et $49 \%$ pour celui de la Madeleine. Seules des mesures comparatives effectuées en ces périodes permettraient de mieux connaître le partage aes débits de la Loire à Nantes.

Quoiqu'il en soit et dans l'état actuel des choses, la proportion des $2 / 3$ et $1 / 3$ dans chacun des deux bras, n'est pas observée. Or, le simple basculement de quelques pourcentages du débit au profit du bras de la Madeleine suffit à modifier la vitesse du courant et à accroitre le pouvoir érosif dans la partie concave des courbes (rapport Sauveterre(9)).

Dans l'état actuel de nos connaissances, il est raisonnable de penser que le partage des débits est sensiblement égal entre les deur bras de la Loire dans leur traversée de Nantes. C'est cette hypothèse qui est désormais retenue pour nos travaux de dynamique sédimentaire.

\section{2. les vitesses}

Si les différences ne sont pas notables pour les volumes oscillants, elles peuvent avoir des conséquences plus importantes sur les vitesses dans chaque bras. Les figures 3 représentant les vitesses en fonction de la profondeur aux points centraux des mesures de 1995 , montrent :

- des profils de vitesse réguliers,

- une légère différence de marnage entre les deux bras. 
En effet, en ce qui conceme ce deuxième point, le bras de la Madieleine est supérieur à celui de Pirmil (environ $1 \mathrm{~m}$ ). Cela peut s'expliquer par les différences dans la largeur des bras et dans la morphologie des berges.

Les courbes de vitesses moyennes pour chaque profil montrent que celles-ci sont supérieures dans le bras de la Madeleine notamment au jusant (voir figures 3 et 4 ). Les volumes sont équivalents et les sections deviennent déterminantes sur les vitesses.

La section et la configuration du bras de la Madeleine sont telles que le secteur est plus sensible à l'érosion que le bras de Pirmil. En période de crue le phénomène est amplifié.
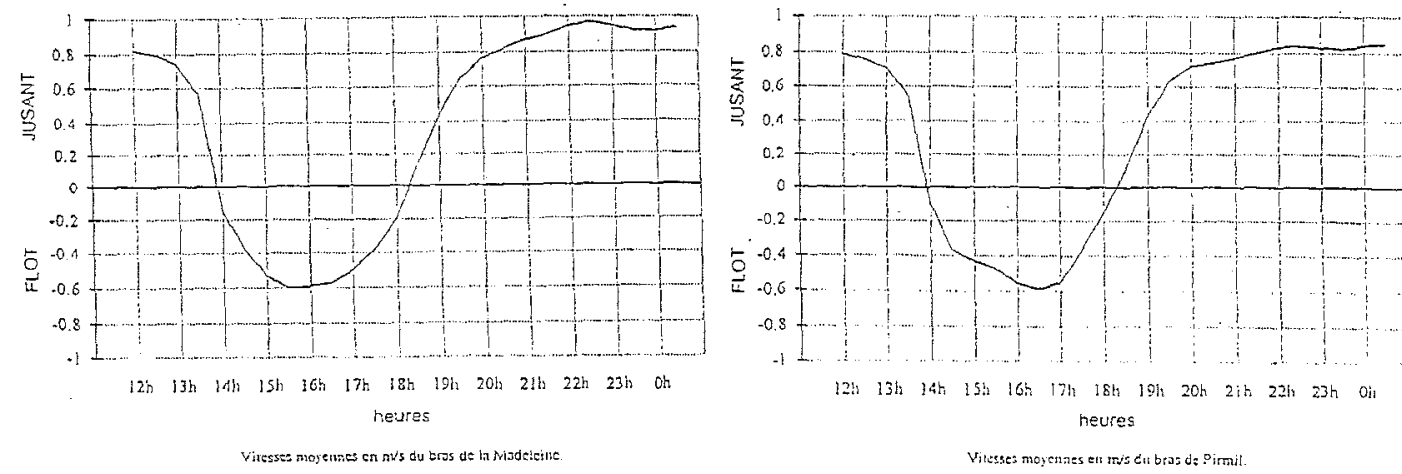

figures 3 : vitesses moyennes en $\mathrm{m} / \mathrm{s}$ dans les bras de La Madeleine et Pirmil (d'après (10)

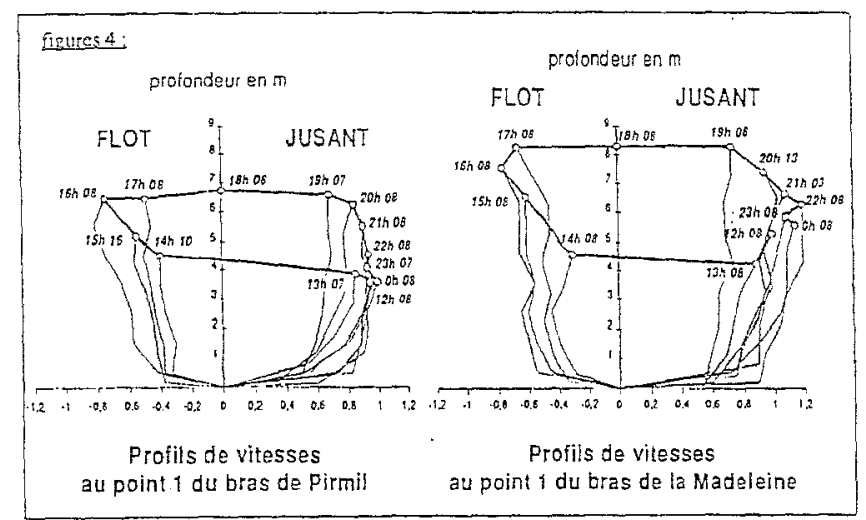

figures 4 : profils de vitesse au flot et au jusant dans le bras de la Madeleine (d'après (10)

\section{Corrélations entre l'analvse hvdrologique et la sédimentologie}

Lors des différentes campagnes, nous avons procèdé à des prélèvements de matières en suspension (M.E.S.) sur toute la colonne d'eau. Que ce soit en juillet 94 ou mai 95, nous n'avons pas localisé le bouchon vaseux; alors qu'en septembre $94\left(240 \mathrm{~m}^{3} / \mathrm{s}\right)$ celui-ci était présent sur notre site d'étude (M.E.S. > $1 \mathrm{~g} / 1$ ). Par 
ailleurs, les prélèvements en profondeur décèlent des concentrations supérieures à $40 \mathrm{~g} /$, ce qui signifie que nous sommes en présence de la crème de vase. Incontestablement, les débits de faible importance favorisent la remontée du bouchon vaseux vers l'amont. Si Galenne(7) constate en 74 que pour un débit équivalent, le bouchon se situe aux alentours du Pellerin, aujourd'hui, c'est au coeur de la ville qu'il se situe.

\subsection{Les courbes d'isoconcentrations}

Les courbes d'isoconcentrations présentées ci-après (figures 5) sont réalisées grâce à la corrélation de la vitesse moyenne des courants sur toute la colonne d'eau et la concentration en MES durant un cycle de marée. Le principe est le suivant: partant d'une renverse qui à lieu à l'heure $\mathrm{Fn}$, on relève le profil de concentration $\mathrm{C}$. Un peu plus tôt à l'heure $\mathrm{Hn}-1$, nous avons relevé le profil de concentration $\mathrm{Cn}-1$. De fait, ce dernier se retrouve à une distance (en aval ou amont selon que l'on se dirige vers le flot ou le jusant):

Dn-Dn-1=(Hn-Hn-1)x vitesse moyenne du courant durant Hn-Hn-1.

On suppose donc qu'il n'y a pas eu de variation verticale de la concentration dans le temps pour une colonne d'eau donnée.

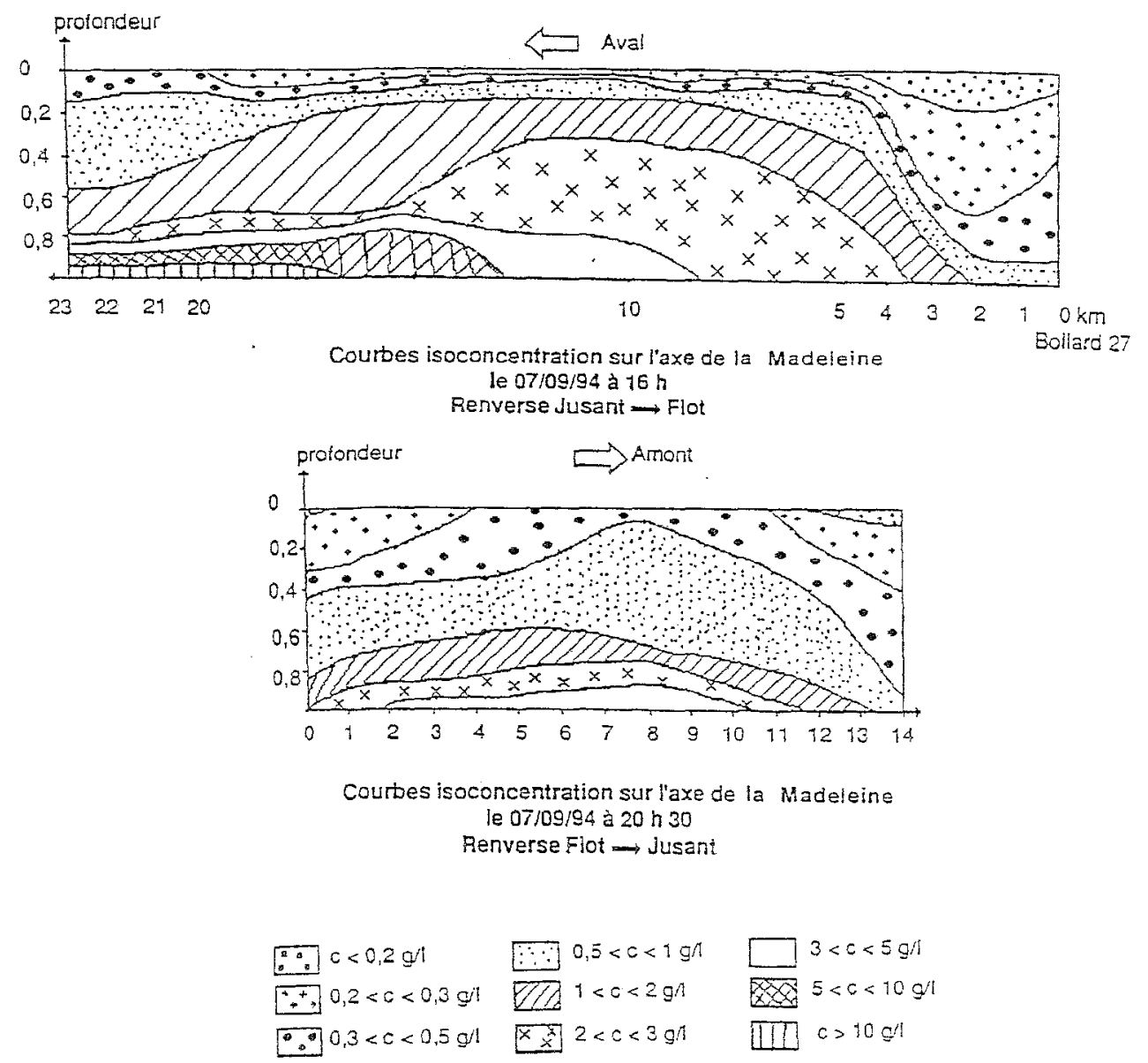

flgures 5 : courbes d'isoconcentration sur l'axe de la Madeleine (d'après (2) 
débits en $\mathrm{m}^{3}$ is

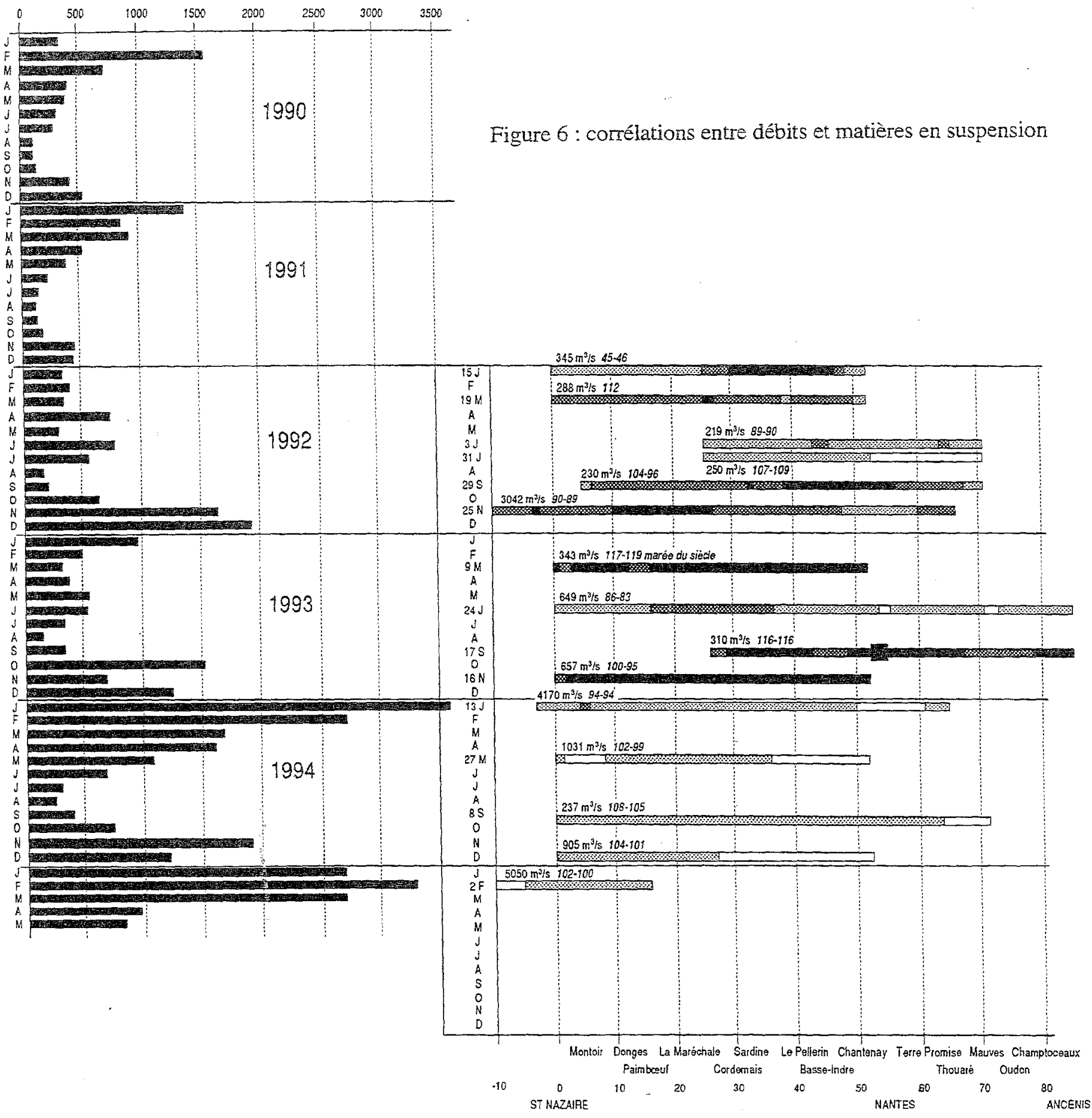

Quantité de matières en suspension

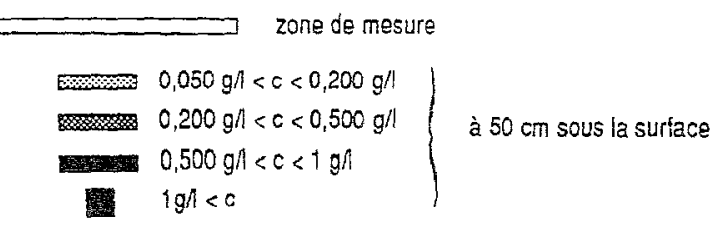




\subsection{Calcul de la masse oscillante}

On estime pour chaque intervalle de concentrations de MES, la proportion qu'il occupe dans la représentation graphique. On a déjà calculé les volumes déplacés de flot et de jusant. Ces volumes, multipliés par les proportions précèdentes donnent les volumes déplacés correspondant à chaque tranche de concentrations.

On calcule la concentration moyenne de chacune des tranches, sachant que la variation de la concentration avec la verticale est approchée par une loi logarithmique. Connaissant volumes et concentrations, on en déduit ainsi la masse oscillante de jusant et de flot.

La masse totale oscillante de jusant dans le bras de la Madeleine pour le 7 septembre 94 est de 52000 tonnes de MES.

Pour le flot, nous avons évalué la masse à 15200 tonnes. A titre de comparaison, en juillet de la mêrne année, nous avons évalué la masse de jusant à 1000 tonnes. Enfin, le même calcul a été effectué en juin 92 pour un débit de $225 \mathrm{~m} 3 / \mathrm{s}$ et la masse au jusant avoisine les 30000 tonnes.

Cinquante deux mille tonnes pour le bras de La Madeleine et l'équivalent pour celui de Pirmil, soit plus de 100000 tonnes transitent le 7 septembre 94 par Nantes. Ceci est considérable et pourtant relativement commun.

La figure 6 présentée ci-contre montre la relation existante entre débits et MES prélevées à $0,5 \mathrm{~m}$ sous la surface de l'eau. Les trois premières années se caractérisent par une faible hydraulicité favorisant les augmentations de concentration des vases. Ce ne sont pas les débits en dents de scie de fin 92 qui permettront de curer le bras. II faut attendre les extrêmes de janvier 94 pour voir enfin la turbidité diminuer.

L'année 1995 a débuté avec des débits de crue étalés sur trois mois et il est probable que les effets sur la qualité des eaux en Loire aient un effet sur le moyen terme.

\section{Conclusions}

Il nous semble intéressant à l'issue de cette étude, d'étendre l'investigation en période de crue (bien que ceci ne soit pas d'une réalisation aisée).

L'étude comparée entre les deux bras, remet en cause l'idée commune d'une répartition du débit de la Loire dans les deux bras selon la loi des 2/3 pour Pirmil et $1 / 3$ pour la Madeleine. En effet, la répartition est plus égale notamment en étiage et cela n'est pas sans conséquences sur la stabilité des berges et d'une façon plus générale sur la dynamique sédimentaire. 


\section{BIBLIOGRAPHTE}

(1) COMITE SCIENTIFQUE POUR L'ENVIRONNEMENT DE L'ESTUAIRE DE LA LOIRE, Rapport final, publication du Centre National pour l'Exploitation des Océans. Rapport scientifique et technique, $\pi^{\circ} 55$, 1984.

(2) ECOLENAVALE ET GROUPE DES ECOLES DE POULMIC, Etude hydrosédimentaire du bras de la Madeleine Projet de fin d'études, Université de Nantes promotion 1992.

(3) P. FATTAL, M. SANCHEZ, V. FREGARD, A. GROVEL, Y. DELANOE, Etude du bras de la Madeieine en aval du pont A. de Bretagne : élargissement du bras et évolution des fonds, Rapport $93-003$ du Centre Français du Littoral, Universitê de Nantes, 1993

(4) P. FATTAL, M. SANCHEZ, A. GROVEL, Y. DELANOE, Problemes d'un estuaire aménagé : la Loire, vers une recherche de solutions concertées, Actes des 3èmes Jounnées Nationales Génie Civil, Génie Côtier, Sète, 2-4 mars 1994 - p. 127 - 132.

(5) P. FATTAL M. ROBEN, Cinématique du lit de la Loire en oval du pont Anne de Bretagne (Nantes) traitée par modélisation numérique de terrain, Colloque Unité de Recherche Associée du C.N.R.S. (U.R.A. 904). 1995,1996, ì paraitre

(6) P. FATTAL, Y. DELANOE, S. ANDRE, Evolution morphologique du lit de lo Loire en aval a"un ouvrage réçent,, Actes des 4 èmes Journées Nationales Génie Civil, Génie Côtier, Dinard 17-19 avril 1996, à paraître

(7) B. GALENNE, Les accumulations turbides dans l'estuaire de la Loire, étude de la crème de vase, thèse de doctorat, Université de Nantes,1974.

(8) J.P. LEVDLLAN, Conséquences de la surexploitation des sables de Loire... Comité Français de Géologie de lingénieur - Mai 1980

(9)SAUVETERRE INGENIEUR CONSEIL, La Loire et le bras de la Madeleine, restauration du patrimoine fluvial, Mairie de Nantes, direction de l'aménagement et de l'urbanisme, janvier 1993

(10) S. TRAN VAN THONG, Comparaison des données hydrologiques entre le bras de Pirmil et de la Madeleine et localisation du bouchon vaseux en 1995. Mémoire de DEA de Génie civil, Génie côtier, soutenu au laboratoire de mécanique et géomécanique de Nantes - Juillet 95 